\title{
Selected Papers from IEEE ICASI 2018
}

\author{
Teen-Hang Meen ${ }^{1, * \mathbb{D}}$, Shoou-Jinn Chang ${ }^{2}$ and Stephen D. Prior ${ }^{3}{ }^{\mathbb{C}}$ \\ 1 Department of Electronic Engineering, National Formosa University, Yunlin 632, Taiwan \\ 2 Department of Electrical Engineering, National Cheng Kung University, Tainan 701, Taiwan; \\ changsj@mail.ncku.edu.tw \\ 3 Aeronautics, Astronautics and Computational Engineering, University of Southampton, \\ Southampton SO16 7QF, UK; S.D.Prior@soton.ac.uk \\ * Correspondence: thmeen@nfu.edu.tw
}

Received: 12 December 2019; Accepted: 13 January 2020; Published: 2 February 2020

check for updates

\begin{abstract}
This Special Issue on "Selected Papers from IEEE ICASI 2018" includes excellent papers presented at the IEEE ICASI 2018 regarding the "applied system innovation" topic. Mechanical engineering and design innovations are both academic and practical engineering fields, which involve systematic technological materialization through scientific principles and engineering designs. Technological innovations in mechanical engineering include IT-based intelligent mechanical systems, mechanics and design innovations, and applied materials in nanosciences and nanotechnology. The aim is to encourage the attendees at the IEEE ICASI 2018 to publish their experimental and theoretical research relating to applied system innovation.
\end{abstract}

Keywords: Smart electromechanical system analysis and design; Intelligent mechanical system; Applied materials on nanosciences and nanotechnology

\section{Introduction}

The 2018 IEEE International Conference on Applied System Innovation (IEEE ICASI 2018) was held in Chiba, Tokyo, Japan, from 13 to 17 April 2018, and provided a unified communication platform for researchers on a wide range of topics. There were more than 700 papers presented in this conference. This Special Issue on "Selected Papers from IEEE ICASI 2018" includes excellent papers presented at the IEEE ICASI 2018 regarding the "applied system innovation" topic. Mechanical engineering and design innovations are both academic and practical engineering fields, which involve systematic technological materialization through scientific principles and engineering designs. Technological innovations in mechanical engineering include IT-based intelligent mechanical systems, mechanics and design innovations, and applied materials in nanosciences and nanotechnology. These new technologies, which implant intelligence into machine systems, are an interdisciplinary area that combines conventional mechanical technologies and new information technologies. The main goal of this Special Issue, "Selected Papers from IEEE ICASI 2018", is to discover new scientific knowledge relevant to IT-based intelligent mechanical systems, mechanics and design innovations, and applied materials in nanosciences and nanotechnology. The aim is to encourage the attendees at the IEEE ICASI 2018 to publish their experimental and theoretical research relating to applied system innovation.

\section{The Topics of Applied System Innovation}

This Special Issue selected 10 excellent papers from 50 papers relevant to the topic of applied system innovation presented at the IEEE ICASI 2018. The published papers are introduced as follows:

Chang et al. reported on "An IP Multimedia Subsystem Services Proxy Gateway Based on a JAVA Dynamic Module System" [1]. This paper proposes an Open Service Gateway Initiative (OSGi) based 
IP multimedia subsystem (IMS) service gateway, in which OSGi technology is the dynamic module system for JAVA. The proposed gateway is authorized by a personal self IMS device such that the IMS services were accessed by non-IMS devices in this paper. Moreover, these non-IMS devices rely on different networks to access technologies and network protocols and the IMS service gateway proposed here adopted the OSGi framework with no problem of integration.

Chang et al. reported on "An Integrated Credit-Based Incentive Protocol for Symbol-Level Network-Coded Cooperative Content Distribution among Vehicular Nodes" [2]. In this paper, based on distinct levels of node interests and selfishness on different content information, the authors, first, categorized vehicular nodes into four classes, that is, destination, intermediate, irrelevant, and overhearing, and then, designated their associated credit-based incentive approaches. Secondly, the authors modified the flow of traditional symbol-level network coding (SLNC) based cooperative content distribution operations and proposed the content bitmap to realize the difference of network-coded content pieces among vehicular nodes. Furthermore, the authors rigidly combined the proposed credit-based incentive approach with the modified SLNC-based cooperative content distribution operations in Social Code to encourage all classes of vehicular nodes to increase their incentives for sharing content data in the cooperative content distribution process. Finally, the authors performed NS-2 simulations on a street map of downtown Taipei, Taiwan and exhibited the high efficiency of Social Code over related credit-based incentive approaches by analyzing the following performance metrics: average decoding percentage, file downloading delay, and credits with respect to different file sizes and total numbers of vehicular nodes.

Chiang et al. reported on "Highly Reliable and Efficient Three-Layer Cloud Dispatching Architecture in the Heterogeneous Cloud Computing Environment" [3]. In this study, a Three-Layer Cloud Dispatching (TLCD) architecture was proposed to enhance the performance of task scheduling. In the first layer, the tasks need to be distinguished into different types by their characters. Subsequently, the cluster selection algorithm is proposed to dispatch the tasks to appropriate service clusters in the second layer. In addition to this, a new scheduling algorithm is proposed in the third layer to dispatch the task to a suitable server in a server cluster to enhance the scheduling efficiency. Basically, the best task completion time was obtained in their TLCD architecture. Furthermore, load balancing and reliability was achieved under a cloud computing network environment.

Cheng et al. reported on "Trajectory Tracking between Josephson Junction and Classical Chaotic System via Iterative Learning Control" [4]. This article addressed trajectory tracking between two nonidentical systems with chaotic properties. To study trajectory tracking, the authors used the Rossler chaotic and resistive-capacitive-inductance shunted Josephson junction (RCLs-JJ) model in a similar phase space. In order to achieve goal tracking, two stages were required to approximate target tracking. The first stage utilized the active control technique to transfer the output signal from the RCLs-JJ system into a quasi-Rossler system. Next, the RCLs-JJ system employed the proposed iterative learning control scheme in which the control signals are from the drive system to trace the trajectory of the Rossler system. The numerical results demonstrated the validity of the proposed method and that the tracking system was asymptotically stable.

Tan et al. reported on "Performance of Resource Allocation in Device-to-Device Communication Systems Based on Evolutionally Optimization Algorithms" [5]. In this study, resource blocks (RB) were allocated to user equipment (UE) according to the evolutional algorithms for long-term evolution (LTE) systems. The particle swarm optimization (PSO) algorithm is one of these evolutionary algorithms, which imitates the foraging behavior of a flock of birds through learning and grouping the best experience. The authors proposed a refined PSO (RPSO) and a novel GA to enhance the throughput of UEs and to improve the system capacity performance. Simulation results showed that the proposed GA with 100 populations coulc converge to suboptimal solutions in 200 generations. The proposed GA and RPSO improved system capacity performance as compared with SPSO by 2.0 and 0.6 UEs, respectively.

Lo reported on the "Application of Aesthetic Principles to the Study of Consumer Preference Models for Vase Forms" [6]. The objective of this study was to investigate consumers' preference 
models from their preferred product forms. The esthetic principles of symmetry, minimalism, and cohesion were applied to the case studies of the design of various vase forms. A quantitative approach of evaluating vase forms by the aesthetic principles was proposed. A conjoint analysis of the vase features and attributes was carried out in order to determine their correlations with consumer preferences. Consumers were classified into six groups of different aesthetic conceptions by cluster analysis. The consumers' preference model could be determined by indices including Pearson's $\mathrm{R}$ and Kendall's tau. This approach provides designers with an effective way of determining the right direction for new form designs and renovation.

Abdul et al. reported on "An Emotion-Aware Personalized Music Recommendation System Using a Convolutional Neural Networks Approach" [7]. In this paper, the authors proposed an emotion-aware personalized music recommendation system (EPMRS) to extract the correlation between the user data and the music. To achieve this correlation, the authors combined the outputs of two approaches, the deep convolutional neural networks (DCNN) approach and the weighted feature extraction (WFE) approach. The DCNN approach is used to extract the latent features from music data (e.g., audio signals and corresponding metadata) for classification. In the WFE approach, the authors generate the implicit user rating for music to extract the correlation between the user data and the music data. For performance comparison, the authors take the content similarity music recommendation system (CSMRS), as well as the personalized music recommendation system based on electroencephalography feedback (PMRSE), as the baseline systems. Experimental results showed that the EPMRS produces better accuracy of music recommendations than the CSMRS and the PMRSE. Moreover, the authors built the Android and iOS APPs to obtain realistic data of user experience on the EPMRS. The collected feedback from anonymous users also showed that the EPMRS sufficiently reflect their preference on music.

Lin et al. reported "Study on Cleaning the Surface of Stainless Steel 316 Using Plasma Electrolysis Technology" [8]. This research utilized a plasma electrolysis technique to clean the surface of stainless steel 316. The resulting microstructure enhances the self-cleaning properties of the stainless steel surface. The position of the cathode electrode is varied to enlarge the total surface being processed and achieves a uniform processing surface. The authors proposed a self-made plasma electrolysis reaction system supplemented with a three-axis platform to control the speed at which the cathode electrode moves. The electrolyte is an aqueous solution of sodium bicarbonate (NaHCO3) and water. The authors obtained the optimal parameters for applied voltage, moving speed of the specimen at the cathode, and electrode distance using a one-factor-at-a-time experimental approach to achieve uniform distribution of the surface microstructure. The contact angle was experimentally proven to be greater than $100^{\circ}$, indicating that the surface was hydrophobic.

Lan et al. reported "Optimization of Machining Parameters Using Fuzzy Taguchi Method for Reducing Tool Wear" [9]. This study investigated the optimal machining parameters for the computer numerical controlled turning process of S45C steel in minimizing tool wear. The correlations between control parameters (speed, cutting depth, and feed rate) and production quality were constructed by using semantic rules and fuzzy quantification. The Taguchi method was additionally employed to determine the optimal turning parameters. Under the consideration of environmental protection and tool cost, the optimal machining parameters were, furthermore, derived from the fuzzy semantic rules. The practicability of the optimal parameters was moreover verified through turning experiments. They found that the proposed method in this study is appropriate and applicable to universal applications.

Chang et al. reported "Finite Element Modeling of an Elderly Person's Cornea and Rigid Gas Permeable Contact Lenses for Presbyopic Patients" [10]. This study adopted finite element modeling to analyze the contact stress between RGP contact lens and an elderly person's cornea. The RGP-lens-produced stress concentration at the corneal edge and maximum pressure on the cornea of elderly subjects aged $>64$ years was $104.140 \mathrm{kPa}$, but only $86.889 \mathrm{kPa}$ for the 15 to 64 age group. Therefore, how to decrease the stress concentration on the cornea is important to increase elderly user comfort while wearing lenses. This study found that when the contact angle was designed on the basis 
of a patient's actual radian of corneal edge, the contact stress dropped sharply to $60.966 \mathrm{kPa}$, thus, increasing the user's wearing comfort.

Author Contributions: Writing and reviewing all papers, T.-H.M.; english editing, S.D.P.; checking and correcting the manuscript, S.J.C. All authors have read and agreed to the published version of the manuscript.

Funding: This research received no external funding.

Acknowledgments: The guest editors would like to thank the authors for their contributions to this special issue and all the reviewers for their constructive reviews. We are also grateful to Emily Zhang, Assistant Managing Editor of Applied Sciences, for her time and efforts on the publication of this special issue for Applied Sciences.

Conflicts of Interest: The authors declare no conflict of interest.

\section{References}

1. Chang, Y.C.; Li, J.W.; Lv, J.H. An IP Multimedia Subsystem Services Proxy Gateway Based on a JAVA Dynamic Module System. Appl. Sci. 2018, 8, 2060. [CrossRef]

2. Chang, I.C.; Yen, C.E.; Lo, J. An Integrated Credit-Based Incentive Protocol for Symbol-Level Network-Coded Cooperative Content Distribution among Vehicular Nodes. Appl. Sci. 2018, 8, 2035. [CrossRef]

3. Chiang, M.L.; Huang, Y.F.; Hsieh, H.C.; Tsai, W.C. Highly Reliable and Efficient Three-Layer Cloud Dispatching Architecture in the Heterogeneous Cloud Computing Environment. Appl. Sci. 2018, 8, 1385. [CrossRef]

4. Cheng, C.K.; Chao, P.C.P. Trajectory Tracking between Josephson Junction and Classical Chaotic System via Iterative Learning Control. Appl. Sci. 2018, 8, 1285. [CrossRef]

5. Tan, T.H.; Chen, B.A.; Huang, Y.F. Performance of Resource Allocation in Device-to-Device Communication Systems Based on Evolutionally Optimization Algorithms. Appl. Sci. 2018, 8, 1271. [CrossRef]

6. Lo, C.H. Application of Aesthetic Principles to the Study of Consumer Preference Models for Vase Forms. Appl. Sci. 2018, 8, 1199. [CrossRef]

7. Abdul, A.; Chen, J.; Liao, H.Y.; Chang, S.H. An Emotion-Aware Personalized Music Recommendation System Using a Convolutional Neural Networks Approach. Appl. Sci. 2018, 8, 1103. [CrossRef]

8. Lin, A.D.; Kung, C.L.; Hsieh, W.C.; Hsu, C.M.; Chen, C.Y. Study on Cleaning the Surface of Stainless Steel 316 Using Plasma Electrolysis Technology. Appl. Sci. 2018, 8, 1060. [CrossRef]

9. Lan, T.S.; Chuang, K.C.; Chen, Y.M. Optimization of Machining Parameters Using Fuzzy Taguchi Method for Reducing Tool Wear. Appl. Sci. 2018, 8, 1011. [CrossRef]

10. Chang, H.C.; Hsu, M.Y.; Hsiao, W.T.; Shum, P.J.T. Finite Element Modeling of an Elderly Person's Cornea and Rigid Gas Permeable Contact Lenses for Presbyopic Patients. Appl. Sci. 2018, 8, 855. [CrossRef]

(C) 2020 by the authors. Licensee MDPI, Basel, Switzerland. This article is an open access article distributed under the terms and conditions of the Creative Commons Attribution (CC BY) license (http://creativecommons.org/licenses/by/4.0/). 